\title{
The Internet, Evolutionary Variational Inequalities, and the Time-Dependent Braess Paradox
}

\section{Citation}

Nagurney, Anna, David C. Parkes, and Patrizia Daniele. 2007. The Internet, evolutionary variational inequalities, and the time-dependent Braess paradox. Computational Management Science 4(4): 355-375.

\section{Published Version}

doi:10.1007/s10287-006-0027-7

\section{Permanent link}

http://nrs.harvard.edu/urn-3:HUL.InstRepos:3996851

\section{Terms of Use}

This article was downloaded from Harvard University's DASH repository, and is made available under the terms and conditions applicable to Other Posted Material, as set forth at http:// nrs.harvard.edu/urn-3:HUL.InstRepos:dash.current.terms-of-use\#LAA

\section{Share Your Story}

The Harvard community has made this article openly available.

Please share how this access benefits you. Submit a story.

\section{Accessibility}


The Internet, Evolutionary Variational Inequalities, and the Time-Dependent Braess Paradox

\author{
Anna Nagurney \\ Radcliffe Institute Fellow \\ Radcliffe Institute for Advanced Study \\ 34 Concord Avenue \\ Harvard University \\ Cambridge, Massachusetts 02138 \\ and \\ Department of Finance and Operations Management \\ Isenberg School of Management \\ University of Massachusetts \\ Amherst, Massachusetts 01003 \\ David Parkes \\ Division of Engineering and Applied Sciences \\ Harvard University \\ Cambridge, Massachusetts 02138 \\ Patrizia Daniele \\ Visiting Scholar \\ Division of Engineering and Applied Sciences \\ Harvard University \\ Cambridge, Massachusetts 02138 \\ and \\ Department of Mathematics and Computer Science \\ University of Catania, Italy
}

May 2006; revised July 2006

\begin{abstract}
In this paper, we develop an evolutionary variational inequality model of the Internet with multiple classes of traffic and demonstrate its utility through the formulation and solution of a time-dependent Braess paradox. The model can handle time-dependent changes in demand as a consequence of developing news stories, following, for example, natural disasters or catastrophes or major media events. The model can also capture the
\end{abstract}


time-varying demand for Internet resources during a regular weekday with its more regular rhythm of work and breaks. In addition, the model includes time-varying capacities on the route flows due to, for example, government interventions or network-type failures. 


\section{Introduction}

The Internet has revolutionized the way in which we work, interact, and conduct our daily activities. It has affected the young and the old as they gather information and communicate and has transformed business processes, financial investing and decision-making, and global supply chains. The Internet has evolved into a network that underpins our developed societies and economies.

In this paper, we develop a dynamic network model of the Internet that is based on evolutionary variational inequality theory. The motivation for this research comes from several directions:

1. The need to develop a dynamic, that is, time-dependent, model of the Internet, as argued, for example, by computer scientists (see Roughgarden (2005)). For example, the demand for Internet resources itself is dynamic and, hence, an underpinning modeling framework must be able to handle time-dependent constraints. Indeed, as noted on page 10 of Roughgarden (2005), "A network like the Internet is volatile. Its traffic patterns can change quickly and dramatically ... The assumption of a static model is therefore particularly suspect in such networks."

2. Analogues have been identified between transportation networks and telecommunication networks and, in particular, the Internet, in terms of decentralized decision-making, flows and costs, and even the Braess paradox, which allows us to take advantage of such a connection (cf. Beckmann, McGuire, and Winsten (1956), Beckmann (1967), Braess (1968), Dafermos and Sparrow (1969), Dafermos (1972), Cantor and Gerla (1974), Gallager (1977), Bertsekas and Tsitsiklis (1989), Bertsekas and Gallager (1992), Korilis, Lazar, and Orda (1999), and Boyce, Mahmassani, and Nagurney (2005)).

3. The development of a fundamental dynamic model of the Internet will allow for the exploration and development of different incentive mechanisms, including dynamic tolls and pricing mechanisms in order to reduce congestion and also aid in the design of a better Internet, a dynamic network, par excellence.

It has been shown (cf. Roughgarden (2005) and the references therein) that distributed 
routing, which is common in computer networks and, in particular, the Internet, and "selfish" (or "source" routing in computer networks) routing, as occurs in the case of user-optimized transportation networks, in which travelers select the minimum cost route between an origin and destination, are one and the same if the cost functions associated with the links that make up the paths/routes coincide with the lengths used to define the shortest paths. In this paper, we assume that the costs on the links are congestion-dependent, that is, they depend on the volume of the flow on the link. Note that the cost on a link may represent travel delay but we utilize "cost" functions since these are more general conceptually than delay functions and they can include, for example, tolls associated with pricing, etc. Of course, it is important to also emphasize that, in the case of transportation networks, it is travelers that make the decisions as to the route selection between origin/destination $(\mathrm{O} / \mathrm{D})$ pairs of nodes, whereas in the case of the Internet, it is algorithms, implemented in software, that determine the shortest paths. Here we assume that these routing algorithms are informed about the cost functions associated with the routes and the volumes of flow on the routes/links in the network and select routes so as to minimize cost. For additional background on telecommunication networks, see Resende and Pardalos (2006).

The methodology that we will utilize for the formulation and analysis of the Internet is that of evolutionary variational inequalities. We believe that such a methodology is quite natural for several reasons. First, historically, finite-dimensional variational inequality theory (cf. Dafermos (1980), Nagurney (1993), and the references therein) has been used to generalize static transportation network equilibrium models dating to the classic work of Beckmann, McGuire, and Winsten (1956), which also forms the foundation for selfish routing and decentralized decision-making on the Internet (see, e.g., Roughgarden (2005)). Secondly, there has been much research activity devoted to the development of models for dynamic transportation problems and it makes sense to exploit the connections between transportation networks and the Internet (see also Nagurney and Dong (2002)). In addition, evolutionary variational inequalities (EVIs), which are infinite-dimensional, have been used to model a variety of time-dependent applications, including time-dependent spatial price problems, financial network problems, dynamic supply chains, and electric power networks (cf. Daniele, Maugeri, and Oettli (1999), Daniele (2003, 2004), Daniele (2006), Nagurney et al. (2006), and Nagurney (2006)). 
In particular, evolutionary variational inequalities (EVIs) were introduced in the 1960s by Brezis (1967) and Lions and Stampacchia (1967), and have been used in the study of partial differential equations and boundary value problems. They are part of the general theory of variational inequalities, which has developed today into a wide-spanning area of research with important applications in control theory, optimization, game theory, operations research, economics, and engineering, notably, in transportation science as well as in logistics (see, for example, Smith (1979), Dafermos (1980), Florian and Los (1982), Dafermos and Nagurney (1984), Nagurney (1989), Zhao and Dafermos (1991), Ran and Boyce (1996), Nagurney and Siokos (1997), and Nagurney (2006), and the references therein). The form of EVI problem that we consider in this paper in a generalization of the one introduced by Daniele, Maugeri, and Oettli (1999) to the case of multiple classes of traffic. We can expect that a variety of time-dependent demand structures will occur on the Internet as individuals seek information and news online in response to major events or simply go about their daily activities whether at work or at home. Hence, the development of a dynamic network model of the Internet is timely. The model that we propose can handle not only time-varying multiclass demands but also time-varying capacities on the multiclass route flows. The latter we can expect to be useful in the case of decreases in capabilities due to network failures or imposed bounds on the Internet traffic on certain routes, due, for example, to policy interventions by governments.

The structure of the paper is as follows. In Section 2 we present the evolutionary variational inequality formulation of the Internet with a focus on the multiclass flows, multiclass costs, and equilibria. In particular, we consider that there are different classes or "jobs" on the Internet and that the equilibrium conditions are associated with each class. We also provide a numerical multiclass dynamic network examples in which the equilibrium trajectories are computed. In Section 3 we illustrate the novelty of this framework in the context of a time-dependent Braess (1968) paradox in which the corresponding evolutionary variational inequalities are explicitly solved. 


\section{Evolutionary Variational Inequalities and the Internet}

We model the Internet as a network $\mathcal{G}=[N, L]$, consisting of the set of nodes $N$ and the set of directed links $L$. The set of links $L$ consists of $n_{L}$ elements. The set of origin/destination (O/D) pairs of nodes is denoted by $W$ and consists of $n_{W}$ elements. We denote the set of routes (with a route consisting of links) joining the origin/destination $(\mathrm{O} / \mathrm{D})$ pair $w$ by $P_{w}$. We assume that the routes are acyclic. We let $P$ with $n_{P}$ elements denote the set of all routes connecting all the $\mathrm{O} / \mathrm{D}$ pairs in the Internet. Links are denoted by $a, b$, etc; routes by $r, q$, etc., and $\mathrm{O} / \mathrm{D}$ pairs by $w_{1}, w_{2}$, etc. We assume that the Internet is traversed by "jobs" or "classes" of traffic and that there are $K$ "jobs" with a typical job denoted by $k$.

Let $d_{w}^{k}(t)$ denote the demand, that is, the traffic generated, between $\mathrm{O} / \mathrm{D}$ pair $w$ at time $t$ by job class $k$. The flow on route $r$ at time $t$ of class $k$, which is assumed to be nonnegative, is denoted by $x_{r}^{k}(t)$ and the flow on link $a$ of class $k$ at time $t$ by $f_{a}^{k}(t)$.

Since the demands over time are assumed known, the following conservation of flow equations must be satisfied at each $t$ :

$$
d_{w}^{k}(t)=\sum_{r \in P_{w}} x_{r}^{k}(t), \quad \forall w \in W, \forall k,
$$

that is, the demand associated with an $\mathrm{O} / \mathrm{D}$ pair and class must be equal to the sum of the flows of that class on the routes that connect that $\mathrm{O} / \mathrm{D}$ pair. We assume that the traffic associated with each O/D pair is divisible and can be routed among multiple routes/paths. Also, we must have that

$$
0 \leq x_{r}^{k}(t) \leq \mu_{r}^{k}(t), \quad \forall r \in P, \forall k,
$$

where $\mu_{r}^{k}(t)$ denotes the capacity on route $r$ of class $k$ at time $t$.

We group the demands at time $t$ of classes for all the O/D pairs into the $K n_{W}$-dimensional vector $d(t)$. Similarly, we group all the class route flows at time $t$ into the $K n_{P}$-dimensional vector $x(t)$. The upper bounds/capacities on the routes at time $t$ are grouped into the $K n_{P}$-dimensional vector $\mu(t)$.

The link flows are related to the route flows, in turn, through the following conservation 
of flow equations:

$$
f_{a}^{k}(t)=\sum_{r \in P} x_{r}^{k}(t) \delta_{a r}, \quad \forall a \in L, \forall k,
$$

where $\delta_{a r}=1$ if link $a$ is contained in route $r$, and $\delta_{a r}=0$, otherwise. Hence, the flow of a class on a link is equal to the sum of the flows of the class on routes that contain that link. All the link flows at time $t$ are grouped into the vector $f(t)$, which is of dimension $K n_{L}$.

The cost on route $r$ at time $t$ of class $k$ is denoted by $C_{r}^{k}(t)$ and the cost on a link $a$ of class $k$ at time $t$ by $c_{a}^{k}(t)$.

For the sake of generality, we allow the cost on a link to depend upon the entire vector of link flows at time $t$, so that

$$
c_{a}^{k}(t)=c_{a}^{k}(f(t)), \quad \forall a \in L, \forall k .
$$

In view of (3), we may write the link costs as a function of route flows, that is,

$$
c_{a}^{k}(x(t)) \equiv c_{a}^{k}(f(t)), \quad \forall a \in L, \forall k
$$

Of course, one special case of (4) would include separable link cost functions in which the cost on a link of a class depends only upon the flow on that link of that class.

The costs on routes are related to costs on links through the following equations:

$$
C_{r}^{k}(x(t))=\sum_{a \in L} c_{a}^{k}(x(t)) \delta_{a r}, \quad \forall r \in P, \forall k
$$

that is, the cost on a route of class $k$ at a time $t$ is equal to the sum of costs on links of that class that make up the route at time $t$. We group the route costs at time $t$ into the vector $C(t)$, which is of dimension $K n_{P}$. Note that the form of (6) also allows such cases as separable route cost functions in which the cost on a route of a class depends only upon the flow of traffic of that class on that route. Furthermore, (6) captures the case in which the cost on a route of a class depends on the total volume of traffic on a route expressed as the sum of the flows of all classes on that route (as well as the sums of flows of the classes on other route(s)). 
We now define the feasible set $\mathcal{K}$. We consider the Hilbert space $\mathcal{L}=L^{2}\left([0, T], R^{K n_{P}}\right)$ (where $[0, T]$ denotes the time interval under consideration) given by

$$
\begin{gathered}
\mathcal{K}=\left\{x \in L^{2}\left([0, T], R^{K n_{P}}\right): 0 \leq x(t) \leq \mu(t) \text { a.e. in }[0, T] ;\right. \\
\left.\sum_{p \in P_{w}} x_{p}^{k}(t)=d_{w}^{k}(t), \forall w, \forall k \text { a.e. in }[0, T]\right\} .
\end{gathered}
$$

We assume that the capacities $\mu_{r}^{k}(t)$, for all $r$ and $k$, are in $\mathcal{L}$, and that the demands, $d_{w}^{k} \geq 0$, for all $w$ and $k$, are also in $\mathcal{L}$. Further, we assume that

$$
0 \leq d(t) \leq \Phi \mu(t), \text { a.e. on }[0, T]
$$

where $\Phi$ is the $K n_{W} \times K n_{P}$-dimensional O/D pair-route incidence matrix, with element $(k w, k r)$ equal to 1 if route $r$ is contained in $P_{w}$, and 0 , otherwise. Here we assume that all classes can use all the routes. (If there are restrictions then the matrix $\Phi$ can be adapted accordingly.) Due to (8), the feasible set $\mathcal{K}$ is nonempty. It is easily seen that $\mathcal{K}$ is also convex, closed, and bounded. Note that we are not restricted as to the form that the time-varying demands for the $\mathrm{O} / \mathrm{D}$ pairs take since convexity of $\mathcal{K}$ is guaranteed even if the demands have a step-wise structure, or are piecewise continuous.

The dual space of $\mathcal{L}$ will be denoted by $\mathcal{L}^{*}$. On $\mathcal{L} \times \mathcal{L}^{*}$ we define the canonical bilinear form by

$$
\langle\langle G, x\rangle\rangle:=\int_{0}^{T}\langle G(t), x(t)\rangle d t, \quad G \in \mathcal{L}^{*}, \quad x \in \mathcal{L} .
$$

Furthermore, the cost mapping $C: \mathcal{K} \rightarrow \mathcal{L}^{*}$, assigns to each flow trajectory $x(\cdot) \in \mathcal{K}$ the cost trajectory $C(x(\cdot)) \in \mathcal{L}^{*}$.

We are now ready to state the dynamic multiclass network equilibrium conditions governing the Internet, assuming shortest path routing. In particular, we assume that the traffic associated with each $\mathrm{O} / \mathrm{D}$ pair and class is selfishly routed to minimize the cost incurred for each class, given the other flows in the network, and subject to the capacity constraints. These conditions are a generalization of the Wardropian (1952) first principle of traffic behavior (see also, e.g., Beckmann, McGuire, and Winsten (1956), Dafermos and Sparrow 
(1969), Dafermos $(1972,1982)$, and Nagurney (1993)) to include multiple classes, the time dimension, and capacities on the route flows. Of course, if the capacities are very large and exceed the demand for a class at each $t$, then the upper bounds are never attained by the route flows and the conditions below will collapse, in the case of fixed time $t$, to the well-known multiclass static network equilibrium conditions (see Dafermos $(1972,1982)$ and the references therein).

\section{Definition 1: Dynamic Multiclass Network Equilibrium}

A multiclass route flow pattern $x^{*} \in \mathcal{K}$ is said to be a dynamic network equilibrium (according to the generalization of Wardrop's first principle) if, at each time $t$, only the minimum cost routes for each class not at their capacities are used (that is, have positive flow) for each $O / D$ pair unless the flow of that class on a route is at its upper bound (in which case those class routes' costs can be lower than those on the routes not at their capacities). The state can be expressed by the following equilibrium conditions which must hold for every $O / D$ pair $w \in W$, every route $r \in P_{w}$, every class $k ; k=1, \ldots, K$, and a.e. on $[0, T]$ :

$$
C_{r}^{k}\left(x^{*}(t)\right)-\lambda_{w}^{k *}(t)\left\{\begin{array}{lll}
\leq 0, & \text { if } & x_{r}^{k *}(t)=\mu_{r}^{k}(t), \\
=0, & \text { if } & 0<x_{r}^{k *}(t)<\mu_{r}^{k}(t), \\
\geq 0, & \text { if } & x_{r}^{k *}(t)=0 .
\end{array}\right.
$$

Hence, conditions (10) state that all utilized routes not at their capacities connecting an $\mathrm{O} / \mathrm{D}$ pair have equal and minimal costs at each time $t$ in $[0, T]$, where that minimal cost is denoted by $\lambda_{w}^{k *}(t)$. If a route flow of a class is at its capacity then its cost can be lower than the minimal cost for that $\mathrm{O} / \mathrm{D}$ pair and class. Of course, if we have that $\mu_{r}^{k}=\infty$, for all routes $r \in P$ and classes $k ; k=1, \ldots, K$, then the dynamic equilibrium conditions state that all used routes connecting an $\mathrm{O} / \mathrm{D}$ pair of nodes for a given class have equal and minimal route costs at each time $t$. For fixed $t$, the latter conditions coincide with a multiclass version of Wardrop's first principle (see Dafermos $(1972,1982)$ ) governing static transportation network equilibrium problems. Note that this concept, but in the case of a single class, has also been applied to static models of the Internet (cf. Roughgarden (2005) and the references therein). We note that El Azouzi (2002) proposed a multiclass network equilibrium model for telecommunications (including the Internet) but the model is 
subsumed by the model of Dafermos (1982). In addition, that model was static. Here, (10) includes the time dimension.

The standard form of the EVI that we work with is:

$$
\text { determine } x^{*} \in \mathcal{K} \text { such that }\left\langle\left\langle F\left(x^{*}\right), x-x^{*}\right\rangle\right\rangle \geq 0, \forall x \in \mathcal{K} \text {. }
$$

We now establish the following theorem.

\section{Theorem 1}

$x^{*} \in \mathcal{K}$ is an equilibrium flow according to Definition 1 if and only if it satisfies the evolutionary variational inequality:

$$
\int_{0}^{T}\left\langle C\left(x^{*}(t)\right), x(t)-x^{*}(t)\right\rangle d t \geq 0, \quad \forall x \in \mathcal{K} .
$$

Proof: We first prove that equilibrium conditions (10) imply the evolutionary variational inequality (12).

Assume that (10) holds. Then

$$
\begin{aligned}
& \sum_{k=1}^{K} \sum_{w \in W} \sum_{r \in P_{w}} C_{r}^{k}\left(x^{*}(t)\right)\left(x_{r}^{k}(t)-x_{r}^{k *}(t)\right) \\
& =\sum_{k=1}^{K} \sum_{w \in W}\left(\sum_{\substack{r \in P_{w} \\
C_{r}^{k}\left(x^{*}(t)\right)>\lambda_{w}^{k *}(t)}} C_{r}^{k}\left(x^{*}(t)\right) x_{r}^{k}(t)+\sum_{\substack{r \in P_{w}^{w} \\
C_{r}^{k}\left(x^{*}(t)\right)=\lambda_{w}^{k *}(t)}} C_{r}^{k}\left(x^{*}(t)\right)\left(x_{r}^{k}(t)-x_{r}^{k *}(t)\right)\right. \\
& \left.+\sum_{\substack{r \in P_{w} \\
C_{r}^{k}\left(x^{*}(t)\right)<\lambda_{w}^{k *}(t)}} C_{r}^{k}\left(x^{*}(t)\right)\left(x_{r}^{k}(t)-\mu_{r}^{k}(t)\right)\right) \\
& \geq \sum_{k=1}^{K} \sum_{w \in W}\left(\sum_{\substack{r \in P_{w} \\
C_{r}^{k}\left(x^{*}(t)\right)>\lambda_{w}^{k *}(t)}} \lambda_{w}^{k *}(t)\left(x_{r}^{k}(t)-x_{r}^{k *}(t)\right)+\lambda_{w}^{k *}(t) \sum_{\substack{r \in P_{w} \\
C_{r}^{k}\left(x^{*}(t)\right)=\lambda_{w}^{k *}(t)}}\left(x_{r}^{k}(t)-x_{r}^{k *}(t)\right)\right.
\end{aligned}
$$




$$
\begin{aligned}
& \left.+\sum_{\substack{r \in P_{w} \\
C_{r}^{k}\left(x^{*}(t)\right)<\lambda_{w}^{k *}(t)}} C_{r}^{k}\left(x^{*}(t)\right)\left(x_{r}^{k}(t)-\mu_{r}^{k}(t)\right)\right) \\
& \geq \sum_{k=1}^{K} \sum_{w \in W}\left(\lambda_{w}^{k *}(t) \sum_{\substack{r \in P_{w} \\
C_{r}^{k}\left(x^{*}(t)\right)>\lambda_{w}^{k *}(t)}}\left(x_{r}^{k}(t)-x_{r}^{k *}(t)\right)+\lambda_{w}^{k *}(t) \sum_{\substack{r \in P_{w} \\
C_{r}^{k}\left(x^{*}(t)\right)=\lambda_{w}^{k *}(t)}}\left(x_{r}^{k}(t)-x_{r}^{k *}(t)\right)\right. \\
& \left.+\lambda_{w}^{k *}(t) \sum_{\substack{r \in P_{w} \\
C_{r}^{k}\left(x^{*}(t)\right)<\lambda_{w}^{k *}(t)}}\left(x_{r}^{k}(t)-x_{r}^{k *}(t)\right)\right) \\
& =\sum_{k=1}^{K} \sum_{w \in W} \lambda_{w}^{k *}(t) \sum_{r \in P_{w}}\left(x_{r}^{k}(t)-x_{r}^{k *}(t)\right)=0 \quad \text { a.e in }[0, T] .
\end{aligned}
$$

Hence, (12) is verified.

We now establish that (12) implies (10). The proof is by contradiction.

First of all let us remark that conditions (10) imply:

$$
\begin{gathered}
\forall k, \forall w \in W, \forall q, s \in P_{w} \text { if } C_{q}^{k}\left(x^{*}(t)\right)<C_{s}^{k}\left(x^{*}(t)\right) \\
\text { then } x_{q}^{k *}(t)=\mu_{q}^{k}(t) \text { or } x_{s}^{k *}(t)=0 .
\end{gathered}
$$

In fact:

1. if $C_{q}^{k}\left(x^{*}(t)\right) \geq \lambda_{w}^{k *}(t)$, then $C_{s}^{k}\left(x^{*}(t)\right)>\lambda_{w}^{k *}(t)$ and $x_{s}^{k *}(t)=0$;

2. if $C_{q}^{k}\left(x^{*}(t)\right)<\lambda_{w}^{k *}(t) \leq C_{s}^{k}\left(x^{*}(t)\right)$, then $x_{q}^{k *}(t)=\mu_{q}^{k}(t)$;

3. if $C_{q}^{k}\left(x^{*}(t)\right)<C_{s}^{k}\left(x^{*}(t)\right)<\lambda_{w}^{k *}(t)$, then $x_{q}^{k *}(t)=\mu_{q}(t)$.

Assume now that equilibrium conditions (10) do not hold. Then, there exists a $k$, a $w \in W$, and routes $q, s \in P_{w}$ together with a set $E \subseteq[0, T]$ having positive measure such that

$$
C_{q}^{k}\left(x^{*}(t)\right)<C_{s}^{k}\left(x^{*}(t)\right), \quad x_{q}^{k *}(t)<\mu_{q}^{k}(t), \quad x_{s}^{k *}(t)>0, \text { a.e. on } E \text {. }
$$


For $t \in E$, let $\delta(t)=\min \left\{\mu_{q}^{k}(t)-x_{q}^{k *}(t), x_{s}^{k *}(t)\right\}$. Then, $\delta(t)>0$ a.e. on $E$, and we can construct $x \in \mathcal{K}$, such that $x^{k}(t)=x^{k *}(t)$ outside $E$ and $x_{q}^{k}(t)=x_{q}^{k *}(t)+\delta(t)$, $x_{s}^{k}(t)=x_{s}^{k *}(t)-\delta(t)$, with $x_{r}^{k}(t)=x_{r}^{k}(t)$, for $r \neq q$, s. Substitution of this feasible flow into $\left\langle\left\langle C\left(x^{*}\right), x-x^{*}\right\rangle\right\rangle$ yields:

$$
\left\langle\left\langle C\left(x^{*}\right), x-x^{*}\right\rangle\right\rangle=\int_{E} \delta(t)\left(C_{q}^{k}\left(x^{*}(t)\right)-C_{s}^{k}\left(x^{*}(t)\right)\right) d t<0,
$$

but this is a contradiction to EVI (12) being satisfied.

\section{Remark 1}

It is important to note that in the proof of Theorem 1 we have, in effect, a dynamic version of the Nash equilibrium concept in that we show that a positive reallocation of $\delta(t)$ of flow from one route to another of a given class will result in a worsened route cost. For a survey of networking games in telecommunications but in a static framework, see Altman et al. (2005). Daniele, Maugeri, and Oettli (1999) presented dynamic network equilibrium conditions for transportation networks but considered only a single class of traffic. Here, we state the dynamic equilibrium conditions in a manner that is more transparent (cf. (10)), noting that the lower bounds on the route flows on the Internet will be zero. In addition, we generalize the results of Daniele, Maugeri, and Oettli (1999) to the case of multiple classes. Finally, the equivalence proof for the EVI formulation is slightly different from that contained in the previous reference, since here we use the equilibrium conditions (10) directly, and also we now have multiple classes of traffic.

We now, for completeness, provide some qualitative properties.

\section{Theorem 2 (cf. Daniele, Maugeri, and Oettli (1999) and Daniele (2006))}

If $C$ in (12) satisfies any of the following conditions:

1. $C$ is hemicontinuous with respect to the strong topology on $\mathcal{K}$, and there exist $A \subseteq \mathcal{K}$ nonempty, compact, and $B \subseteq \mathcal{K}$ compact such that, for every $y \in \mathcal{K} \backslash A$, there exists $x \in B$ with $\langle\langle C(x), y-x\rangle\rangle<0$;

2. $C$ is hemicontinuous with respect to the weak topology on $\mathcal{K}$; 
3. $C$ is pseudomonotone and hemicontinuous along line segments,

then the EVI problem (12) admits a solution over the constraint set $\mathcal{K}$.

Recall that $C: \rightarrow \mathcal{L}^{*}$, where $\mathcal{K}$ is convex, is said to be

pseudomonotone if and only if, for all $x, y \in \mathcal{K}$

$$
\langle\langle C(x), y-x\rangle\rangle \Rightarrow\langle\langle C(y), x-y\rangle\rangle \leq 0
$$

hemicontinuous if and only if, for all $y \in \mathcal{K}$, the function $\xi \mapsto\langle\langle C(\xi), y-\xi\rangle$ is upper semicontinuous on $\mathcal{K}$;

hemicontinuous along line segments if and only if, for all $x, y \in \mathcal{K}$, the function $\xi \mapsto$ $\langle\langle C(\xi), y-x\rangle\rangle$ is upper semicontinuous on the line segment $[x, y]$.

Moreover, if $C$ is strictly monotone, then the solution of (12) is unique (see, e.g., Kinderlehrer and Stampacchia (1980)).

\subsection{A Multiclass Numerical Example}

We now present a small multiclass dynamic network equilibrium numerical example.

Consider a network (small subnetwork of the Internet) consisting of two nodes and two links as in Figure 1. There is a single O/D pair $w=(1,2)$. Since the routes connecting the $\mathrm{O} / \mathrm{D}$ pair consist of single links we work with the routes $r_{1}$ and $r_{2}$ directly as in Figure 1 .

There are assumed to be two classes/jobs and the route costs are:

for Class 1:

$$
C_{r_{1}}^{1}(x(t))=2 x_{r_{1}}^{1}(t)+x_{r_{1}}^{2}(t)+5, \quad C_{r_{2}}^{1}(x(t))=2 x_{r_{2}}^{2}(t)+2 x_{r_{2}}^{1}(t)+10,
$$

for Class 2:

$$
C_{r_{1}}^{2}(x(t))=x_{r_{1}}^{2}(t)+x_{r_{1}}^{1}(t)+5, \quad C_{r_{2}}^{2}(x(t))=x_{r_{2}}^{1}(t)+2 x_{r_{2}}^{2}(t)+5 .
$$




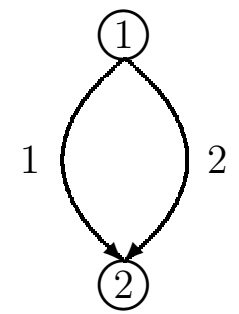

Figure 1: Network Structure of the Multiclass Numerical Example

The time horizon is $[0,10]$. The demands for the O/D pair are:

$$
d_{w}^{1}(t)=10-t, \quad d_{w}^{2}(t)=t
$$

The upper bounds are: $\mu_{r_{1}}^{1}=\mu_{r_{2}}^{1}=\mu_{r_{1}}^{1}=\mu_{r_{2}}^{2}=\infty$.

To solve the associated evolutionary variational inequality, we utilize the approach set forth in Cojocaru, Daniele, and Nagurney (2005 a, b), in which the time horizon $T$ is discretized and the corresponding variational inequality (or, equivalently, projected dynamical system) at each discrete point in time is then solved. Due to the simplicity of the network structure, we can easily obtain such solutions by solving the equilibrium conditions (10) explicitly at the discrete time points.

Obviously, this procedure is correct if the continuity of the solution is guaranteed. Continuity results for solutions to evolutionary variational inequalities, in the case where $F(x(t))=$ $A(t) x(t)+B(t)$ is a linear operator, $A(t)$ is a continuous and positive definite matrix in $[0, T]$, and $B(t)$ is a continuous vector can be found in Barbagallo (2005). Of course, the examples could also be computed via the computational procedure given in Daniele, Maugeri, and Oettli (1999) but here we utilize a time-discretization approach which also has intuitive appeal. In Table 1 we provide the equilibrium solutions for the multiclass network equilibrium example at discrete points in time.

In Figure 2, we provide a graph of the equilibrium route trajectories, where we display also the interpolations between the discrete solutions given in Table 1. Since the route cost functions are strictly monotone over the time horizon $[0,10]$ we know that the equilibrium trajectories are unique. Moreover, as the theory predicts, the trajectories are also continuous 
Table 1: Equilibrium Route Flows for the Multiclass Numerical Example

\begin{tabular}{|c|c|c|c|c|c|}
\hline \multicolumn{5}{|c|}{ Equilibrium Multiclass Route Flows at time $t$} \\
\hline Flow & $t=0$ & $t=2.5$ & $t=5$ & $t=7.5$ & $t=10$ \\
\hline$x_{r_{1}}^{1 *}(t)$ & 6.25 & 6.25 & 5.00 & 2.50 & 0.00 \\
$x_{r_{2}}^{1 *}(t)$ & 3.75 & 1.25 & 0.00 & 0.00 & 0.00 \\
$x_{r_{1}}^{2 *}(t)$ & 0.00 & 0.00 & $1 . \overline{66}$ & $4.1 \overline{66}$ & $6 . \overline{66}$ \\
$x_{r_{2}}^{2 *}(t)$ & 0.00 & 2.50 & $3 . \overline{33}$ & $3 . \overline{33}$ & $3 . \overline{33}$ \\
\hline
\end{tabular}

for this example. It is interesting to see that after time $t=5$ route $r_{2}$ is never used by class 1 , whereas route $r_{1}$ is not utilized for class 2 traffic until after $t=2$.

For completeness, we also provide the following class $\mathrm{O} / \mathrm{D}$ pair minimum costs at times $t=0,2.5,5,7.5$ and 10 :

$\lambda_{w}^{1 *}(0)=17.50, \quad \lambda_{w}^{1 *}(2.5)=17.50, \quad \lambda_{w}^{1 *}(5)=16 . \overline{6}, \quad \lambda_{w}^{1 *}(7.5)=14.1 \overline{66}, \quad \lambda_{w}^{1 *}(10)=11 . \overline{6} 6$

and

$$
\lambda_{w}^{2 *}(0)=8.75, \quad \lambda_{w}^{2 *}(2.5)=11.25, \quad \lambda_{w}^{2 *}(5)=11 . \overline{66}, \quad \lambda_{w}^{2 *}(7.5)=11 . \overline{66}, \quad \lambda_{w}^{2 *}(10)=11 . \overline{6} .
$$

\section{Remark 2}

It is easy to verify that if the first cost term in $C_{r_{1}}$ is changed from "2" to "1" then there are multiple equilibria. 


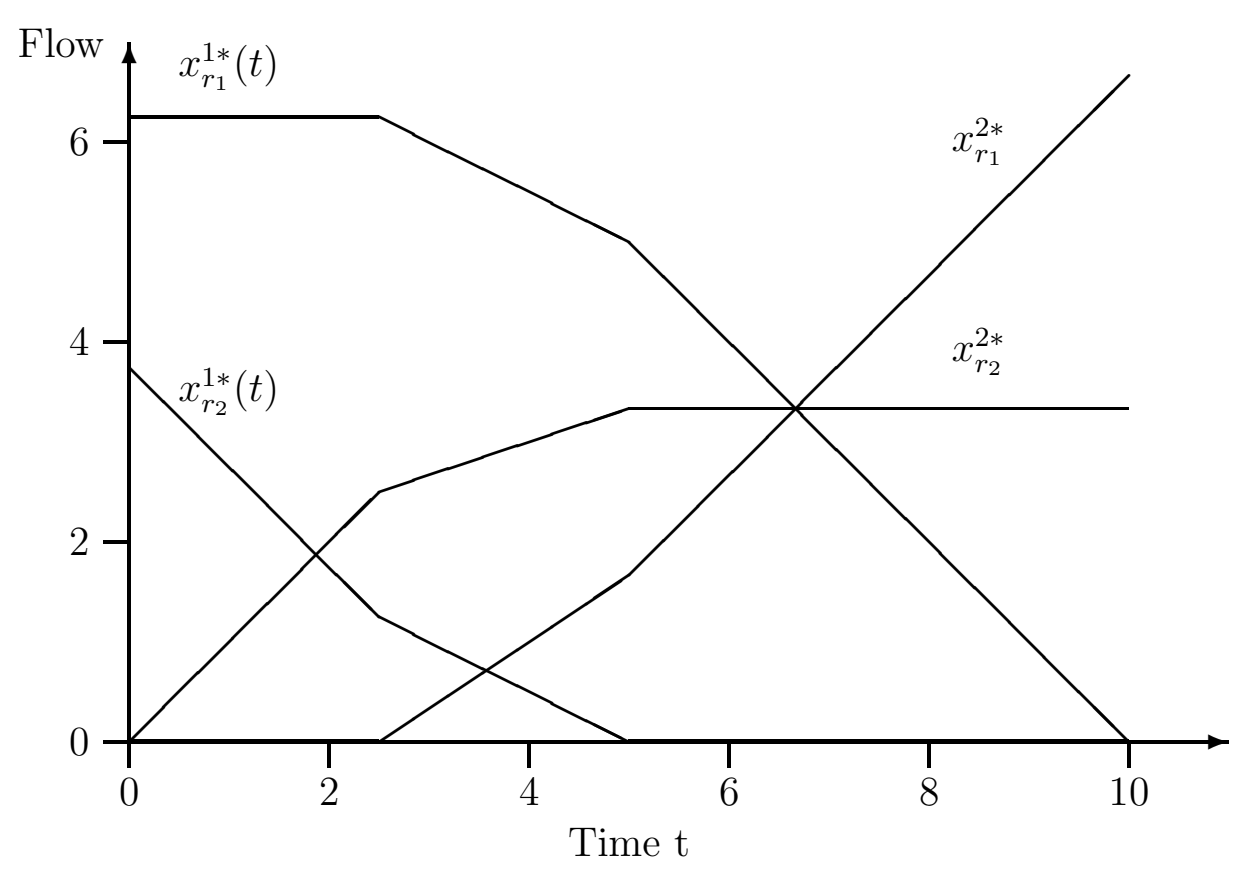

Figure 2: Equilibrium Trajectories for the Multiclass Numerical Example

\section{Evolutionary Variational Inequalities and the Braess Paradox}

We further reinforce the elegance and power of the evolutionary variational inequality model for the Internet as we revisit the Braess (1968) paradox (see also, Boyce, Mahmassani, and Nagurney (2005) and Braess, Nagurney, and Wakolbinger (2005)). Examples of the Braess paradox, originally formulated for transportation networks, have occurred in cities such as New York as well as Stuttgart. In addition, the Braess paradox has been noted to occur not only in transportation networks but also in telecommunication networks, including the Internet.

Recall that in the Braess paradox, which is an example of a fixed demand network equilibrium problem, the addition of a new link, which yields a new route, makes all the "users" in the network worse off. As emphasized in Korilis, Lazar, and Orda (1999), this is also relevant to the Internet. We present an evolutionary variational inequality formulation which deepens the understanding of the Braess paradox and also illustrates dramatically the importance of time-varying demands and the associated equilibrium flows and costs in what 

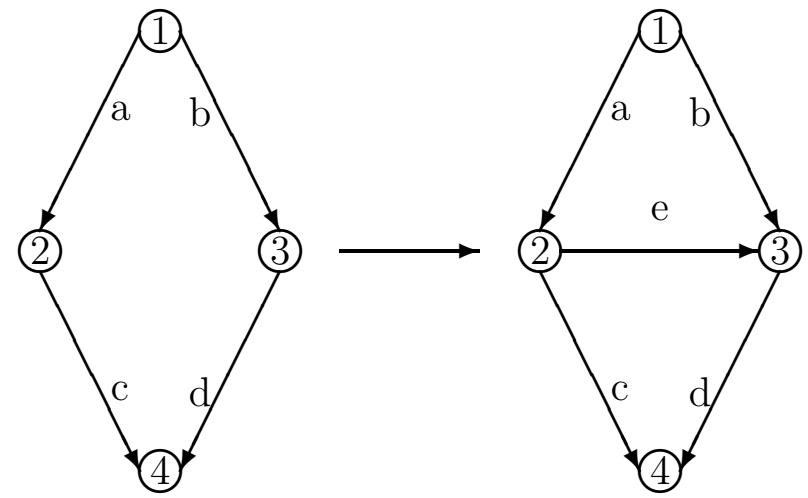

Figure 3: The Time-Dependent Braess Network Example with Relevance to the Internet

are increasingly becoming known as noncooperative networks. We assume, hence, that there is a single class $k$ and we supress the superscript $k$ in the notation below.

\section{The Time-Dependent Braess Paradox}

Assume a network as the first network depicted in Figure 3 in which there are four nodes: $1,2,3,4$; four links: $a, b, c, d$; and a single $\mathrm{O} / \mathrm{D}$ pair $w=(1,4)$. There are, hence, two routes available between this $\mathrm{O} / \mathrm{D}$ pair: $r_{1}=(a, c)$ and $r_{2}=(b, d)$.

The networks given in Figure 3 are due to Braess (1968). We now construct timedependent link costs, route costs, and demand for $t \in[0, T]$. It is important to emphasize that the case where time $t$ is discrete, that is, $t=0,1,2, \ldots, T$, is trivially included in the equilibrium conditions (10) and also captured in the evolutionary variational inequality formulation (12).

We consider, to start, the first network in Figure 3, consisting of links: $a, b, c, d$. We assume that the capacities $\mu_{r_{1}}(t)=\mu_{r_{2}}(t)=\infty$ for all $t \in[0, T]$. The link cost functions are assumed to be given and as follows for time $t \in[0, T]$ :

$$
\begin{aligned}
& c_{a}\left(f_{a}(t)\right)=10 f_{a}(t), \quad c_{b}\left(f_{b}(t)\right)=f_{b}(t)+50, \\
& c_{c}\left(f_{c}(t)\right)=f_{c}(t)+50, \quad c_{d}\left(f_{d}(t)\right)=10 f_{d}(t) .
\end{aligned}
$$


We assume a time-varying demand $d_{w}(t)=t$ for $t \in[0, T]$.

Observe that at time $t=6, d_{w}(6)=6$, and it is easy to verify that the equilibrium route flows at time $t=6$ are:

$$
x_{r_{1}}^{*}(6)=3, \quad x_{r_{2}}^{*}(6)=3,
$$

the equilibrium link flows are:

$$
f_{a}^{*}(6)=3, \quad f_{b}^{*}(6)=3, \quad f_{c}^{*}(6)=3, \quad f_{d}^{*}(6)=3,
$$

with associated equilibrium route costs:

$$
C_{r_{1}}(6)=c_{a}(6)+c_{c}(6)=83, \quad C_{r_{2}}=c_{b}(6)+c_{d}(6)=83,
$$

and, hence, equilibrium condition (10) is satisfied for time $t=6$. This is the solution to the classical (static) Braess (1968) network without the route addition.

We now construct and solve EVI (12) for the dynamic network equilibrium problem over $t \in[0, T]$. We first express the route costs in terms of route flows for Network 1 in Figure 3, where we have that, because of the conservation of flow equations $(3), f_{a}(t)=f_{c}(t)=x_{r_{1}}(t)$ and $f_{b}(t)=f_{d}(t)=x_{r_{2}}(t)$. That is, we must have that

$$
C_{r_{1}}(t)=11 x_{r_{1}}(t)+50, \quad C_{r_{2}}(t)=11 x_{r_{2}}(t)+50,
$$

with the route conservation of flow equations (1) yielding:

$$
d_{w}(t)=t=x_{r_{1}}(t)+x_{r_{2}}(t)
$$

and, hence, we may write

$$
x_{r_{2}}(t)=t-x_{r_{1}}(t)
$$

Similarly, we must have, because of the feasible set $\mathcal{K}$ (cf. (7)), the simplicity of the network topology, and the cost structure, that

$$
x_{r_{1}}^{*}(t)=x_{r_{2}}^{*}(t)
$$

Hence, we may write EVI (12) for this problem as: determine $x^{*} \in \mathcal{K}$ satisfying

$$
\int_{0}^{T}\left(11 x_{r_{1}}^{*}(t)+50\right) \times\left(x_{r_{1}}(t)-x_{r_{1}}^{*}(t)\right)+\left(11 x_{r_{2}}^{*}(t)+50\right) \times\left(x_{r_{2}}(t)-x_{r_{2}}^{*}(t)\right) d t \geq 0, \quad \forall x \in \mathcal{K},
$$


which, in view of (16), can be expressed as:

$$
\int_{0}^{T}\left(11 x_{r_{1}}^{*}(t)+50\right) \times\left(x_{r_{1}}(t)-x_{r_{1}}^{*}(t)\right)+\left(11\left(t-x_{r_{1}}^{*}(t)\right) \times\left(x_{r_{1}}(t)-x_{r_{1}}^{*}(t)\right) d t \geq 0, \quad \forall x \in \mathcal{K},\right.
$$

which, after algebraic simplification, is

$$
\int_{0}^{T}\left(22 x_{r_{1}}^{*}(t)-11 t\right) \times\left(x_{r_{1}}(t)-x_{r_{1}}^{*}(t)\right) d t \geq 0, \quad \forall x \in \mathcal{K} .
$$

But, (19) implies that:

$$
22 x_{r_{1}}^{*}(t)=11 t ; \text { for } t \in[0, T]
$$

or

$$
x_{r_{1}}^{*}(t)=\frac{t}{2} .
$$

Hence, we also have that $x_{r_{2}}^{*}(t)=\frac{t}{2}$.

Moreover, the equilibrium route costs for $t \in[0, T]$ are given by:

$$
C_{r_{1}}\left(x_{r_{1}}^{*}(t)\right)=5 \frac{1}{2} t+50=C_{r_{2}}\left(x_{r_{2}}^{*}(t)\right)=5 \frac{1}{2} t+50,
$$

and, clearly, equilibrium conditions (10) hold for $\in[0, T]$ a.e.

Assume now that, as depicted in Figure 3, a new link " $e$ ", joining node 2 to node 3 is added to the original network, with cost $c_{e}\left(f_{e}(t)\right)=f_{e}(t)+10$ for $t \in[0, T]$. The addition of this link creates a new route $r_{3}=(a, e, d)$ that is available for the Internet traffic. Assume that the time-varying demand is still given by $d_{w}(t)=t$. Note, that for $t=6$, for example, the original equilibrium flow distribution pattern $x_{r_{1}}(6)=3$ and $x_{r_{2}}(6)=3$ is no longer an equilibrium pattern, since at this level of flow the cost on route $r_{3}, C_{r_{3}}(6)=70$. Hence, the traffic from routes $r_{1}$ and $r_{2}$ would be switched to route $r_{3}$.

The equilibrium flow pattern at time $t=6$ on the new network (which would correspond to the classic Braess paradox in a static network equilibrium setting) is:

$$
x_{r_{1}}^{*}(6)=2, \quad x_{r_{2}}^{*}(6)=2, \quad x_{r_{3}}^{*}(6)=2,
$$

with equilibrium link flows:

$$
f_{a}^{*}(6)=4, \quad f_{b}^{*}(6)=2, \quad f_{c}^{*}(6)=2, \quad f_{e}^{*}(6)=2, \quad f_{d}^{*}(6)=4
$$


and with associated equilibrium route costs:

$$
C_{r_{1}}(6)=92, \quad C_{r_{2}}(6)=92, \quad C_{r_{3}}(6)=92
$$

Indeed, one can verify that any reallocation of the route flows would yield a higher cost on a route.

Note that, with the route addition, the cost at time $t=6$ increased for every "user" of the network from 83 to 92 without a change in the demand or traffic rate! This is the classical Braess paradox.

We now solve the evolutionary variational inequality problem (12) for the second network in Figure 3 over the time interval $[0, T]$ to create the time-dependent Braess paradox.

We may write the route costs for the second network (after the route addition) in Figure 3 as a function of the time-dependent route flows, that is,

$$
C_{r_{1}}(x(t))=11 x_{r_{1}}(t)+10 x_{r_{3}}(t)+50, \quad C_{r_{2}}(x(t))=11 x_{r_{2}}(t)+10 x_{r_{3}}(t)+50,
$$

and

$$
C_{r_{3}}(x(t))=10 x_{r_{1}}(t)+21 x_{r_{3}}(t)+10 x_{r_{2}}(t)+10 .
$$

EVI (12) now takes the form: determine $x^{*} \in \mathcal{K}$ (where $\mathcal{K}$ is now expanded to include route $r_{3} ;$ see $\left.(7)\right)$, so that

$$
\begin{array}{r}
\int_{0}^{T}\left(11 x_{r_{1}}^{*}(t)+10 x_{r_{3}}^{*}(t)+50\right) \times\left(x_{r_{1}}(t)-x_{r_{1}}^{*}(t)\right)+\left(11 x_{r_{2}}^{*}(t)+10 x_{r_{3}}^{*}(t)+50\right) \times\left(x_{r_{2}}(t)-x_{r_{2}}^{*}(t)\right) \\
+\left(10 x_{r_{1}}^{*}(t)+21 x_{r_{3}}^{*}(t)+10 x_{r_{2}}^{*}(t)+10\right) \times\left(x_{r_{3}}-x_{r_{3}}^{*}(t)\right) d t \geq 0, \quad \forall x \in \mathcal{K} . \quad(20)
\end{array}
$$

Because of the feasible set $\mathcal{K}$, we must have that

$$
x_{r_{3}}(t)=t-x_{r_{1}}(t)-x_{r_{2}}(t) \text { and } x_{r_{3}}^{*}(t)=t-x_{r_{1}}^{*}(t)-x_{r_{2}}^{*}(t) .
$$

Substitution of (21) into (20), after algebraic simplification, yields

$$
\int_{0}^{T}\left(x_{r_{1}}^{*}(t)-11\left(t-x_{r_{1}}^{*}(t)-x_{r_{2}}^{*}(t)\right)-10 x_{r_{2}}^{*}(t)+40\right) \times\left(x_{r_{1}}(t)-x_{r_{1}}^{*}(t)\right)
$$


$+\left(x_{r_{2}}^{*}(t)-11\left(t-x_{r_{1}}^{*}(t)-x_{r_{2}}^{*}(t)\right)-10 x_{r_{2}}^{*}(t)+40\right) \times\left(x_{r_{2}}(t)-x_{r_{2}}^{*}(t)\right) d t \geq 0, \quad \forall x \in \mathcal{K}$.

Now, since it is clear (because of the network topology and cost structure) that $x_{r_{1}}^{*}(t)=$ $x_{r_{2}}^{*}(t)$ we can simplify EVI (22) further to:

$$
\int_{0}^{T}\left(13 x_{r_{1}}^{*}(t)-11 t+40\right) \times\left(\left(x_{r_{1}}(t)+x_{r_{2}}(t)\right)-2 x_{r_{1}}^{*}(t)\right) d t \geq 0, \quad \forall x \in \mathcal{K} .
$$

We now analyze (23). In particular, we consider the term:

$$
\left(13 x_{r_{1}}^{*}(t)-11 t+40\right) \times\left(x_{r_{1}}(t)+x_{r_{2}}(t)-2 x_{r_{1}}^{*}(t)\right)
$$

for a fixed $t$ and analyze when its value is greater than or equal to zero. We note that if $x_{r_{1}}^{*}(t)=0$, then for this term to be greater than or equal to zero, we must have that $-11 t+40 \geq 0$, or $t \in\left[0,3 \frac{7}{11}\right]$. We, hence, obtain that:

$$
x_{r_{1}}^{*}(t)=x_{r_{2}}^{*}(t)=0, \quad x_{r_{3}}^{*}(t)=t, \text { for } t \in\left[0,3 \frac{7}{11}\right] .
$$

On the other hand, if $x_{r_{1}}^{*}(t)>0$, we must consider the situation that either $x_{r_{3}}^{*}(t)$ is also greater than zero or it is equal to zero. We first consider the case that $x_{r_{3}}^{*}(t)=0$. Then, we know that $x_{r_{1}}^{*}(t)=x_{r_{2}}^{*}(t)=\frac{t}{2}$. Substitution of this expression for $x_{r_{1}}^{*}(t)$ into $(24)$, states that this value is valid for $t \in\left(8 \frac{1}{9}, \infty\right)$.

In the range for $t \in\left(3 \frac{7}{11}, 8 \frac{8}{9}\right]$ we obtain that:

$$
x_{r_{1}}^{*}(t)=x_{r_{2}}^{*}(t)=\frac{11}{13} t-\frac{40}{13}, \quad x_{r_{3}}^{*}(t)=-\frac{9}{16} t+\frac{43}{8} .
$$

\section{Remark 3}

For both networks in Figure 3, with the associated link and route cost functions, it is easy to verify that the corresponding vector of route costs $C(x)$ is strictly monotone in route flows $x$, that is,

$$
\left\langle\left\langle C\left(x^{1}\right)-C\left(x^{2}\right), x^{1}-x^{2}\right\rangle\right\rangle>0, \quad \forall x^{1}, x^{2} \in \mathcal{K}, x^{1} \neq x^{2},
$$


since the Jacobian of the route costs is strictly diagonally dominant at each $t$ and, thus, positive definite. Hence, the corresponding equilibrium route flow solutions $x^{*}(t)$ will be unique.

In summary, we have identified three regimes, denoted by I, II, and III, respectively, and depicted in Figure 4, where: for $d_{w}(t)=t \in\left[0, t_{1}=3 \frac{7}{11}\right]$ (Regime I):

$$
x_{r_{1}}^{*}(t)=x_{r_{2}}^{*}(t)=0, \quad x_{r_{3}}^{*}(t)=d_{w}(t)=t ;
$$

for $d_{w}(t)=t \in\left(t_{1}=3 \frac{7}{11}, 8 \frac{8}{9}\right]$ (Regime II), we have that:

$$
x_{r_{1}}^{*}(t)=x_{r_{2}}^{*}(t)=\frac{11}{13} t-\frac{40}{13}, \quad x_{r_{3}}^{*}(t)=-\frac{9}{16} t+\frac{43}{8} .
$$

Finally, for $d_{w}(t)=t \in\left(t_{2}=8 \frac{8}{9}, T<\infty\right]$ (Regime III), we have that:

$$
x_{r_{1}}^{*}(t)=x_{r_{2}}^{*}(t)=\frac{d_{r_{1}}(t)}{2}=\frac{t}{2}, \quad x_{r_{3}}^{*}(t)=0 .
$$

The curves of equilibria are depicted in Figure 4.

Clearly, one can see from Figure 4 , that in the range $\left(0, t_{1}=3 \frac{7}{11}\right]$, that is, in Regime I (once the demand is positive), only the new route $r_{3}$ would be used. Hence, at a relatively low level of demand, up to a value of $3 \frac{7}{11}$, only the new route is used. In the range of demands: $\left(3 \frac{7}{11}, 8 \frac{8}{9}\right]$, that is, Regime II, all three routes are used, and in this range the Braess paradox occurs. Finally, once the demand (recall that $d_{w}(t)=t$ here) exceeds $8 \frac{8}{9}$ and we are in Regime III, then the new route is never used! Thus, the use of an evolutionary variational inequality formulation reveals that over time the Braess paradox is even more profound and the addition of a new route may result in the route never being used. Finally, if the demand lies within a particular range, then the addition of a new route may result in everyone being worse off, since it results in higher costs than before the route/link was added to the network.

In particular, the "classical" Braess paradox, in which the addition of the route makes the "travel" cost higher for everyone, always occurs in Regime II. In order to find the minimal 


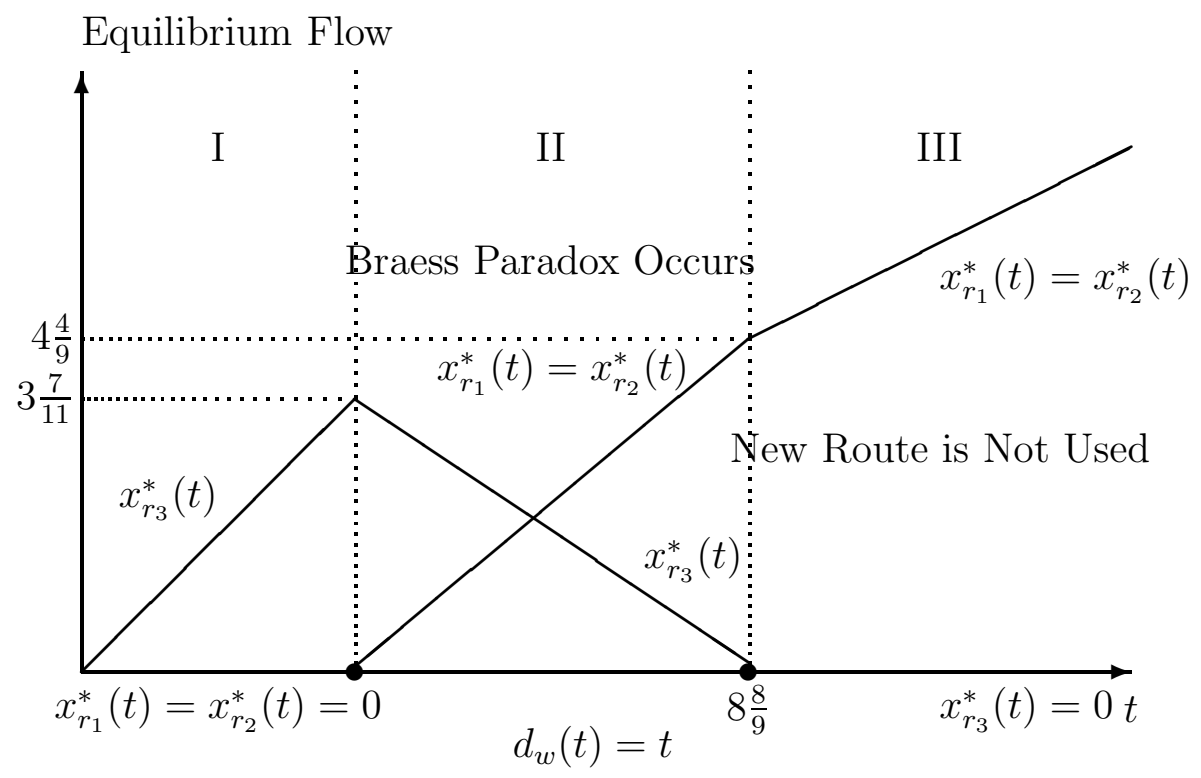

Figure 4: Equilibrium Trajectories of the Braess Network with Time-Dependent Demands

demand at which the Braess paradox occurs, we note that in the first network in Figure 3, the demand will always equally distribute itself. Hence, on the original network, the equilibrium flow pattern on each route would be given by $\frac{d_{w}(t)}{2}=\frac{t}{2}$ for $t \in[0, T]$ with a minimal route cost over the horizon being, thus, equal to: $11\left(\frac{t}{2}\right)+50$. Consider now, the second network in Figure 3. We know that in Regime I, only the new route would be used, assuming shortest path routing, with the minimal route cost, hence, being given by the expression in this range of demands as $21 t+10$. Setting now, $11\left(\frac{t}{2}\right)+50=21 t+10$, and solving for $t$, which, is also in this problem equal to the demand, $d_{w}(t)$, yields $t=2 \frac{18}{31}=2.58$. For demand in the range $2.58<d_{w}(t)=t<8 \frac{8}{9}=8.89$, the addition of the new route will result in everyone being worse off. See Figure 5 .

Pas and Principio (1997) obtained precisely this result but using a static formulation and in the context of transportation networks. The evolutionary variational inequality formulation provides a compact form for uncovering the time-dependent paradoxical results. Moreover, since the vector of route costs is strictly monotone, as argued above, we know that the solution to the evolutionary variational inequality (12) is unique and so the curve of 


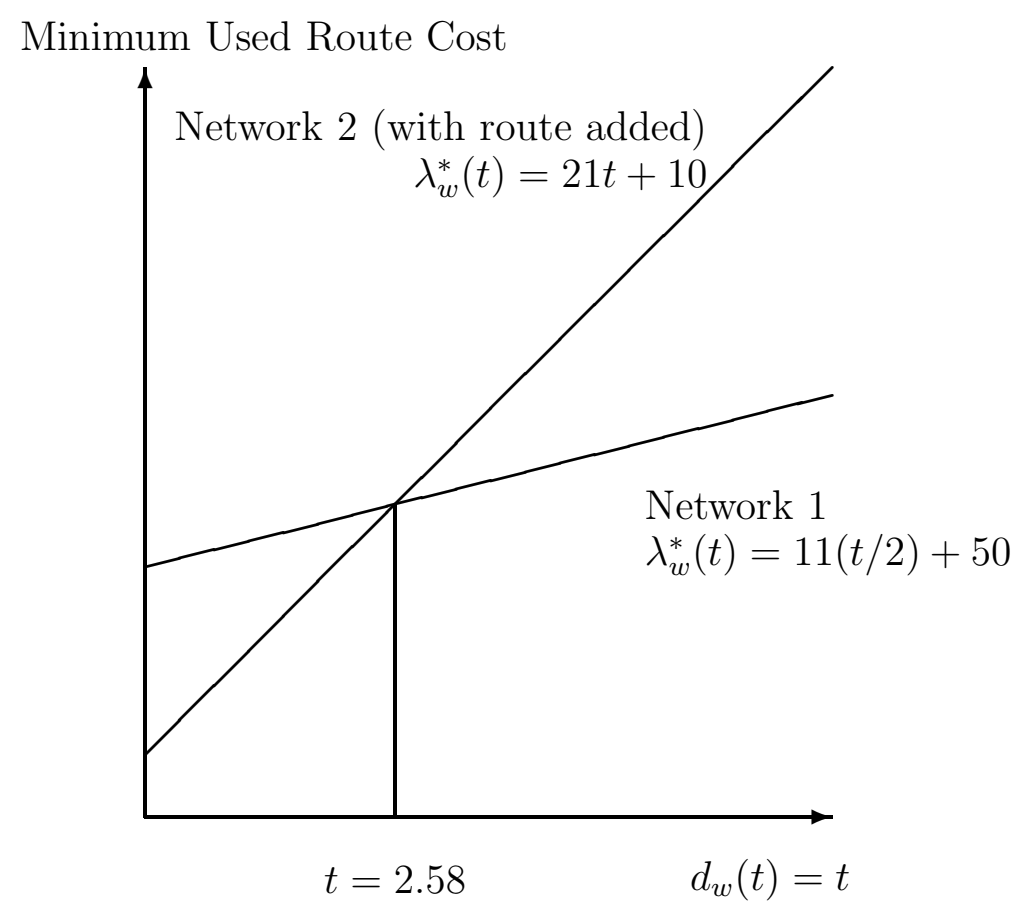

Figure 5: Minimum Used Route Costs for Networks 1 and 2 
equilibria is unique, as depicted in Figure 4. Furthermore, we can see, as the theory predicts, that the equilibrium trajectories (cf. Figure 4) are continuous.

Nagurney (2006) also presented this time-dependent Braess paradox but in the context of dynamic transportation network equilibrium problems. Here, we provide the complete, explicit, solution of the EVI formulations for Network 1 and for Network 2. Arnott, De Palma, and Lindsey (1993), motivated by the Braess paradox, presented a paradox in the

context of a dynamic transportation network equilibrium problem in which the routes are fixed but users decide when to travel, and, hence, the demand is also dynamic. Their focus was, however, on queues and expanding capacity in a particular network and did not make use of evolutionary variational inequality theory.

\section{Acknowledgments}

The first author acknowledges support from the Radcliffe Institute for Advanced Study at Harvard University and from NSF Grant No. IIS-0002647.

The third author acknowledges support from Harvard University while she was a Visiting Scholar in the Division of Engineering and Applied Sciences for the spring semester in 2006. The support provided is gratefully acknowledged.

The authors acknowledge helpful discussions with Professor Antonino Maugeri while Patrizia Daniele was a Visiting Scholar at DEAS at Harvard and for his assistance and support of this research and special support provided by Dean Barbara Grosz of the Radcliffe Institute for Advanced Study and DEAS at Harvard University. The authors would also like to thank Zugang "Leo" Liu for assistance with the first numerical example. 


\section{References}

Altman, E., Boulogne, T., El Azouzi, R., Jimenez, T., and Wynter, L. (2005), A Survey on Networking Games in Telecommunications, INRIA, Cedex, France.

Arnott, R., De Palma, A., and Lindsey, R. (1993), Properties of Dynamic Traffic Equilibrium Involving Bottlenecks, Including a Paradox and Metering, Transportation Science 27, 148160.

Barbagallo, A. (2005), Regularity Results for Time-Dependent Variational Inequalities and Quasi-Variational Inequalities and Computational Procedures, to appear in Mathematical Models and Methods in Applied Sciences.

Barbagallo A. (2006), Regularity Results for Time-dependent Degenerate Variational Inequalities and Applications to Time-dependent Equilibrium Problems, to appear in Computational Methods in Applied Mathematics 6.

Beckmann, M. J.(1967) On the Theory of Traffic Flows in Networks, Traffic Quarterly 21, 109-116.

Beckmann, M. J., McGuire, C. B., and Winsten, C. B. (1956) Studies in the Economics of Transportation, Yale University Press, New Haven, Connecticut.

Bertsekas, D. P., and Gallager, R. G. (1987) Data Networks, Prentice-Hall, Englewood Cliffs, New Jersey.

Bertsekas, D. P., and Tsitsiklis, J. N. (1997) Parallel and Distributed Computation: Numerical Methods, Prentice-Hall, Englewood Cliffs, New Jersey.

Boyce, D.E., Mahmassani, H. S., and Nagurney, A. (2005) A Retrospective of Beckmann, McGuire, and Winsten's Studies in the Economics of Transportation, Papers in Reional Science 84, 85-103.

Braess, D. (1968) Uber ein Paradoxon aus der Verkehrsplaning, Unternehmenforschung 12, $258-268$.

Braess, D., Nagurney, A., and Wakolbinger, T. (2005) On a Paradox of Traffic Planning, 
Translation of the 1968 Article by Braess, Transportation Science 39, 446-450.

Brezis, H. (1967) Inequations D'Evolution Abstraites, Comptes Rendue d'Academie des Sciences.

Cantor, D. G., and Gerla, M. (1974) Optimal Routing in a Packet-Switched Computer Network, IEEE Transactions on Computers 23, 1062-1069.

Cojocaru, M.-G., Daniele, P., and Nagurney, A. (2005a) Projected Dynamical Systems and Evolutionary Variational Inequalities, Journal of Optimization Theory and Applications 27, $1-15$.

Cojocaru, M.-G., Daniele, P., and Nagurney, A. (2005b) Double-Layered Dynamics: A Unified Theory of Projected Dynamical Systems and Evolutionary Variational Inequalities, European Jurnal of Operational Research, in press.

Dafermos, S. C. (1972) The Traffic Assignment Problem for Multiclass-User Transportation Networks, Transportation Science 6, 73-87.

Dafermos, S. (1980) Traffic Equilibrium and Variational Inequalities, Transportation Science 14, 42-54.

Dafermos, S. (1982) The General Multimodal Network Equilibrium Problem with Elastic Demand, Networks 12, 57-72.

Dafermos, S. and Nagurney, A. (1984) Sensitivity Analysis for the General Spatial Economic Equilibrium Problem, Operations Research 32, 1069-1086

Dafermos, S. C., and Sparrow, F. T. (1969) The Traffic Assignment Problem for a General Network, Journal of Research of the National Bureau of Standards 73B, 91-118.

Daniele, P. (2003) Evolutionary Variational Inequalities and Economic Models for Demand Supply Markets, Mathematical Models and Methods in Applied Sciences 4, 471-489.

Daniele, P. (2004) Time-Dependent Spatial Price Equilibrium Problem: Existence and Stability Results for the Quantity Formulation Model, Journal of Global Optimization 28, 283295. 
Daniele, P. (2006) Dynamic Networks and Evolutionary Variational Inequalities, Edward Elgar Publishing, Cheltenham, England.

Daniele, P., Maugeri, A., and Oettli, W. (1999) Time-Dependent Variational Inequalities, Journal of Optimization Theory and its Applications 103, 543-555.

El Azouzi, R. (2002) Avoiding Paradox in Routing Games in Networks when Travel Demand is Elastic, Proceedings of the Tenth Symposium on Dynamic Games and Applications, St. Petersburg, Russia.

Florian, M., and Los, M. (1982) A New Look at Static Spatial Price Equilibrium Models, Regional Science and Urban Ecnomics 12, 579-597.

Gallager, R. G. (1977) A Minimum Delay Routing Algorithm Using Distributed Computation, IEEE Transaction on Communications 25, 73-85.

Kinderlehrer, D., and Stampacchia, G. (1980) An Introduction to Variational Inequalities and their Applications, Academic Press, New York.

Korilis, Y. A., Lazar, A. A., and Orda, A. (1999) Avoiding the Braess Paradox in NonCooperative Networks, Journal of Applied Probability 36, 211-222.

Lions, J. L., and Stampacchia, G. (1967) Variational Inequalities, Communications in Pure and Applied Mathematics 22, 493-519.

Nagurney, A. (1989) Migration Equilibrium and Variational Inequalities, Economics Letters 31, 109-112.

Nagurney, A. (1993) Network Economics: A Variational Inequality Approach, Kluwer Academic Publishers, Dordrecht, The Netherlands.

Nagurney, A. (2006) Supply Chain Network Economics: Dynamics of Prices, Flows, and Profits, Edward Elgar Publishing, Cheltenham, England.

Nagurney, A., and Dong, J. (2002) Supernetworks: Decision-Making for the Information Age, Edward Elgar Publishing, Cheltenham, England.

Nagurney, A., Liu, Z., Cojocaru, M. -G., and Daniele, P. (2006) Static and Dynamic Trans- 
portation Network Equilibrium Reformulations of Electric Power Supply Chain Networks with Known Demands, Transportation Research E, in press.

Nagurney, A., and Siokos, S. (1997) Financial Networks: Statics and Dynamics, Springer Verlag, Heidelberg, Germany.

Pas, E. I., and Principio, S. L. (1997), Braess Paradox: Some New Insights, Transportation Research B 31, 265-276.

Ran, B., and Boyce, D. E. (1996) Modeling Dynamic Transportation Networks, Springer Verlag, Berlin, Germany.

Resende, M. G. C., and Pardalos, P. M., Editors (2006) Handbook of Optimization in Telecommunications, Springer Science and Business Media, New York.

Roughgarden, T. (2005) Selfish Routing and the Price of Anarchy, MIT Press, Cambridge, Massachusetts.

Smith, M. (1979) Existence, Uniquness, and Stability of Traffic Equilibria, Transportation Research B 13, 259-304.

Wardrop, J. G. (1952) Some Theoretical Aspects of Road Traffic Research, Proceedings of the Institute of Civil Engineers, Part II, pp. 325-378.

Zhao, L., and Dafermos, S. (1991) General Economic Equilibrium and Variational Inequalities, Operations Research Letters 10, 369-376. 